\title{
A High Performance Target Tracing Transmission Model Oriented to Lifecycle Maximization
}

\author{
Zhong-Nan Zhao, Pei-Li Qiao, and Jian Wang \\ School of Computer Science and Technology, Harbin University of Science and Technology, Harbin, Heilongjiang 150080, China \\ Correspondence should be addressed to Zhong-Nan Zhao; piconet@126.com
}

Received 21 March 2016; Accepted 10 October 2016

Academic Editor: Fanli Meng

Copyright ( 2016 Zhong-Nan Zhao et al. This is an open access article distributed under the Creative Commons Attribution License, which permits unrestricted use, distribution, and reproduction in any medium, provided the original work is properly cited.

For the high speed sensor networks applications such as Internet of Things, multimedia transmission, the realization of high-rate transmission under limited resources has become a problem to be solved. A high speed transmission and energy optimization model oriented to lifecycle maximization is proposed in this paper. Based on information-directed mechanism, the energy threshold set and the relay node distance selection will be done in the process of target tracing, as a result, retaining a balance between transmission rate and energy consumption. Meanwhile, multiagent coevolution is adopted to achieve the maximum of network lifecycle. Comparing with the relevant methods, indexes for network such as hops, throughput, and number of active nodes, standard deviation of remaining energy, and the network lifecycle are considered, and the simulated experiments show that the proposed method will promote the transmission rate effectively, prolong the network lifecycle, and improve network performance as a whole.

\section{Introduction}

The wireless sensor network (WSN) is a kind of multihop ad hoc network which is composed of a large number of tiny sensor nodes by means of wireless communications $[1,2]$. The function of wireless sensor network is perception, acquisition, and processing of the information of monitored object in the coverage area, and then the information is sent to the observer. The network is widely used in military, intelligent transportation, environmental monitoring, health care, and so forth [3-6]. Due to the small size of sensor nodes, as a result, the energy of battery carried is limited. When sensor nodes are deployed in complex areas, how to optimize the network energy consumption and increase the network lifecycle is one of the key issues. Studies have focused on energy-efficient optimization of wireless sensor network, and some remarkable results such as minimizing the energy for routing and maximum lifecycle have been involved [7-10].

Nowadays facing real-time requirements of the new high speed sensor networks, such as wireless multimedia sensor networks and industrial real-time control, energy conservation cannot meet the needs of practical application completely. The high speed sensor network, as an emerging network, is to advance the traditional sensor network technology in the application of multimedia transmission, and so forth, with higher bandwidth and more computing and processing power, which has become a hotspot in recent years [11, 12]. Compared to the traditional networks, the wireless sensor network has a higher demand in aspects of throughput, realtime, computational complexity, power requirements, and so on. The most essential difference between them lies in throughput requirement which is the basis for high speed sensor network applications. Therefore how to meet the requirements of transmission performance while reducing energy consumption has become an urgent problem.

For wireless sensor networks, the issues of energy saving and lifecycle have been concerned. Literature [13] reveals the findings of lifecycle and predictive perception space in the route selection, in which the differences of node number and perception scale are used with linear programming equations 
to design the different scenarios to promote the lifecycle of networks by computing the normalization. But this method considers the single factor of lifecycle rather than the others. Literature [14] designs a cross-layer unified optimum convex model based on the integration of physics layer, MAC (Media Access Control) layer, and network layer, thus prolonging the network lifecycle. Literature [15] provides a dynamic tuning scheme for the transmission scope of sensor nodes against the emergence of energy hole, which adopts centralized algorithm and distributed algorithm to regulate the transmission scale, thus reducing the complexity of inquiry and promoting the lifecycle. Literature [16] predicts the next location of target-closed routing based on the information of target tracing, in which the nodes located adopt a method of inducing tracking to lead a distant node to move close to the center, thus completing local coverage positioning and tracking the energy consumption. All above studies of lifecycle for the sensor networks are based on the limited resources of sensor network, which do not consider the impact of transmission rate on the system, thus failing to meet with the application requirement of real-time high performance transmissions.

In this paper, some corresponding researches have been done under the background above; at the same time multiagent is used to optimize system. Literature [17] provides a simulation platform of multiagent oriented structure through formal modeling, on which the perception and rationalization can be made by decision-making and action prediction of agents, and the cooperation mechanism of each node can be simulated. Literature [18] analyzes the cooperation and competition relations of wireless sensor networks available for resource task and information processing, by utilizing the characters of multiagent such as distribution and being autonomous and self-organizing. The studies above only modeled and analyzed the network structure based on the multiagent properties, but not the systematic operation. Literature [19] focuses on the confliction and precision of target tracing sensors for the wireless sensor networks. Literature [20] designs a data convergence system with mobile multiagent for the wireless sensor networks, thus obtaining the effective convergence of time and energy to meet with users. The above two use the multiagent technology to realize target tracing and information convergence of networks but did not consider the transmission performance of data. Literature [21] proposed a multiagent energy balance strategy based on two different indicators in cases of wireless sensor networks hotspot being frequently inquired. Literature [22] introduced multiagent to realize the resource distribution in the course of task scheduling, on which the resource distribution and task were heavily correlative. Literature [23] introduces multiagent to locate the target and classify the task and uses the support vector machines to reduce communication load and optimized the system. The studies above analyze the transmission performance on resource distribution, energy consumption, and load, respectively, but did not consider the indicators comprehensively. All the results above are about the use of agent in wireless sensor network, they are all from the perspectives of the similarity of the network structures to formalize modeling or define unit individual, and the issue of system optimization by multiagent has not been considered.

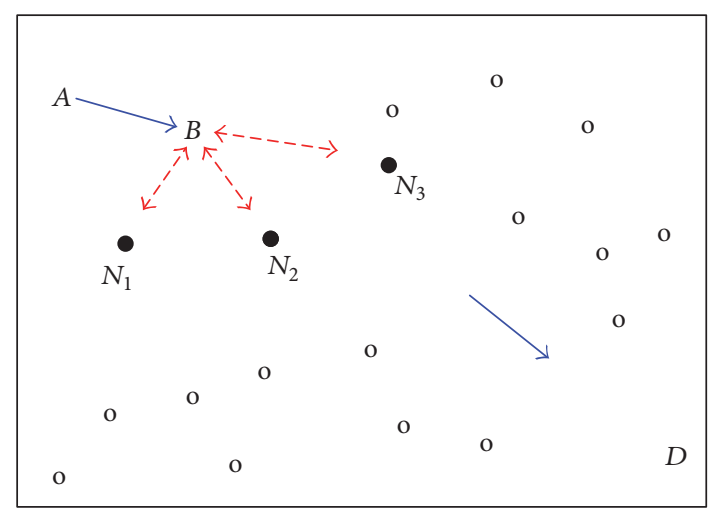

FIGURE 1: The process of target tracing.

As to the problems above, this paper proposes a high speed transmission and energy optimization model oriented to lifecycle maximization (HS-EO), which tracks targets in the way that is information-directed, and selects the relay nodes and sets the energy threshold based on the wireless transmission performance, then gives a link selection based on multiagent coevolution algorithm, and finally realizes the maximum lifecycle of networks while high performance transmission is achieved.

The structure of this paper is organized as follows: Section 2 refers to the problem presentation; Section 3 presents the principles and schemes of HS-EO; Section 4 proposes a multiagent coevolution algorithm based on HS-EO; Section 5 carries on the correlative experimentations and analyses; Conclusions go last.

\section{Problem Descriptions}

HS-EO model is based on the information-directed mechanism and aiming at achieving high throughput and energy optimized transmission in the process of target tracing. Information-directed way can help to trace the target in wireless sensor network without knowing the location of the target node in advance; only some relevant information of the target node is needed. In this process, it is needed to obtain and update information with its neighbor nodes by information exchange, shown in Figure 1. A denotes the start node, $B$ denotes the relay node, and $D$ denotes the target node. While $B$ selects the next hop, it needs to transmit information to the adjacent nodes, namely, $N_{1}, N_{2}$, and $N_{3}$, and then it goes on close to $D$. There are two conditions to be met: one is that the correlative information of target node can be perceived and obtained in wireless sensor networks; the other is that each node can have its own coordinate by GPS or anchors.

The route that is information-directed rests on routing strategies. HS-EO is designed to increase the transmission rate and reduce the energy consumption, in which the relay node selection of high performance transmission is shown in Figure 2.

For node $B$, the radius $r^{*}$ which is able to achieve maximum throughput capacity within its communication radius 


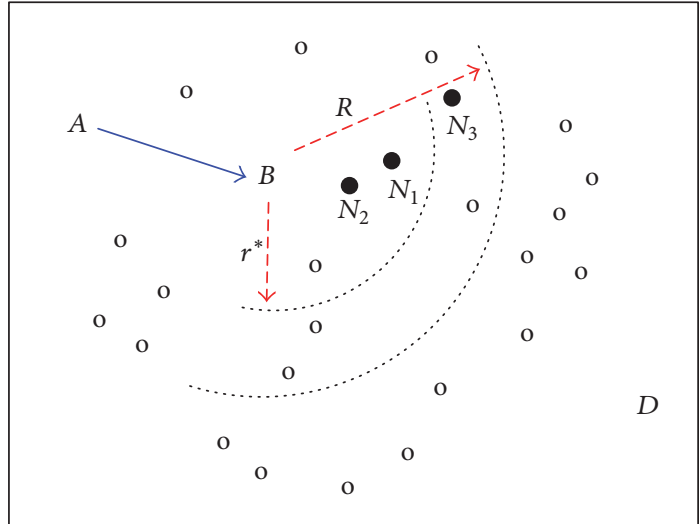

FIGURE 2: Description of radius $r^{*}$.

$R$ can be obtained, and the nodes $N_{1}, N_{2}, N_{3}$ around it can provide high speed rate as well. In order to select high speed nodes while considering energy consumption, a multiagent coevolution algorithm is adopted to optimize the multiple objectives in this paper.

\section{HS-EO Model Definition}

In the process of target tracing, HS-EO is a high performance transmission model which is information-directed. For the problem of routing selection in data transmission, nodes are scheduled through energy threshold setting, and further the nodes with high throughput are selected.

3.1. Information-Directed. Since the information-directed way is suitable for the data transmission in wireless networks, it is often used to make the target location and tracking [24]. In the two-dimensional plane, the position information of the target node can be obtained by a series of measures which use sequence Bayesian filter on a set of nodes $\overline{z^{(t)}}=\left\{z^{(0)}, z^{(1)}\right.$, $\left.\ldots, z^{(t)}\right\}$. Based on the prior information of the target node at the time $t$, the probability density function $p\left(x^{(t)} \mid \overline{z^{(t)}}\right)$ is used to obtain the estimates $z^{(t+1)}$ at time $t+1$. Among them, the sequence Bayesian filter can measure and update $p\left(x^{(t+1)} \mid \overline{z^{(t+1)}}\right)$ through the following formula:

$$
\begin{aligned}
& p\left(x^{(t+1)} \mid \overline{z^{(t+1)}}\right) \\
& \propto p\left(z^{(t+1)} \mid x^{(t+1)}\right) \\
& \quad \cdot \int p\left(x^{(t+1)} \mid x^{(t)}\right) \cdot p\left(x^{(t)} \mid \overline{z^{(t)}}\right) d x^{(t)} .
\end{aligned}
$$

To quantify the contribution of each sensor node, the "Mutual Information (MI)" can be used, which can define the information contribution to the evaluation of relevant nodes. Using the random variable $U, V$, and joint probability density function $p(u, v)$, then the MI can be defined as follows:

$$
\begin{aligned}
\operatorname{MI}(U ; V) & \triangleq E_{p(u, v)}\left[\log \frac{p(u, v)}{p(u) p(v)}\right] \\
& =D(p(u \mid v) \| p(u)),
\end{aligned}
$$

where $\operatorname{MI}(U ; V)$ shows the relationship between $U$ and $V$, which explains how much information that $V$ transfers about $U$. In sequential Bayesian filter, the contribution of measurement $Z_{k}^{(t+1)}$ for node $k$ at the time $t+1$ can be expressed as follows:

$$
I_{\mathrm{MI}, k}=\mathrm{MI}\left(X^{(t+1)} ; Z_{k}^{(t+1)} \mid \overline{Z^{(t)}}=\overline{z^{(t)}}\right) .
$$

Formula (3) shows how much information about location information of the target node $X^{(t+1)}$ is offered by $Z_{k}^{(t+1)}$, so $I_{\mathrm{MI}, k}$ reflects the variation of a priori information generated by node $k$. Thus, if $I_{\mathrm{MI}, k} \neq 0$, this means node $k$ can continue to approach to the direction of target node.

\subsection{Selection of Relay Nodes}

3.2.1. Communication Distance Analysis. In multihop wireless sensor networks, the data are often forwarded by the adjacent nodes, so the relationship between throughput and transmission distance needs to be analyzed [25]. Assuming that the transmission power of the node is fixed, a half-duplex communication mode is adopted, and the location of the nodes in the region obeys the random Poisson distribution. When a node begins transmission, if the node coordinate is taken as the origin, during the transmission, the position of other nodes can be denoted as a set of location coordinates $\Phi$, which is defined as follows:

$$
\Phi=\left\{X_{i} \mid \exists t \in[\tau, \tau+T]: e_{i}(t)=1\right\} \backslash\{0\},
$$

where $[\tau, \tau+T]$ represents the time period of transmission, $e_{i}(t)$ represents the nodes transmitted successfully in $[\tau, \tau+$ $T]$, and $\{0\}$ represents the coordinate origin.

Under the condition of $|Y|=y$, the probability of successful transmission from the origin to a node $Y$ can be expressed as follows:

$$
\begin{aligned}
P_{s \mid y} & \approx \frac{1}{1+\lambda T} e^{-\lambda T} \cdot \operatorname{Pr}\left\{G_{X^{(1)} Y} \leq \frac{G_{0 Y}}{\gamma}-\frac{N}{P}\right\} \\
& =\frac{1}{1+\lambda T} e^{-\lambda T} \cdot \int_{l(u) \leq l(y) / \gamma-N / P} f_{\left|X^{(1)}-Y\right|}(u) d u .
\end{aligned}
$$

According to the definition of the transmission of wireless networks, the intensity of throughput can be defined as follows:

$$
S_{t}=E_{Y}\left[S_{|Y|}|Y| \cos (\arg (Y))\right]
$$

where $|Y| \cos (\arg (Y))$ represents the effective traversal distance to the destination node in single hop.

There are two factors that need to be considered in selecting the next relay node: one is distance, and the other is throughput. Therefore, the throughput intensity needs to be calculated under the condition of distance $y$, as shown in formula (7). Among them, $\gamma$ represents the minimum SINR 
(Signal to Interference Plus Noise Ratio) value required to receive the data.

$$
\begin{aligned}
S_{t \mid y}= & E_{Y}\left[S_{|Y|}|Y| \cos (\arg (Y))|| Y \mid=y\right] \\
= & \exp \left[-\pi v\left(1-\frac{1}{1+\lambda T} e^{-\lambda T}\right)\left(y^{-\alpha} \mid \gamma-\frac{N}{P}\right)^{-2 / \alpha}\right] \\
& \cdot y \cdot E_{Y}[\cos (\arg (Y))] .
\end{aligned}
$$

According to the derivation for formula (7), the maximum throughput $s^{*}$ and the corresponding transmission distance $r^{*}$ can be obtained, shown as follows:

$$
\begin{aligned}
r^{*}= & \left(2 \pi v\left(1-\frac{1}{1+\lambda T} e^{-\lambda T}\right)\right)^{-1 / 2} \gamma^{-1 / \alpha} \\
s^{*}= & b \cdot\left(2 \pi v\left(1-\frac{1}{1+\lambda T} e^{-\lambda T}\right)\right)^{-1 / 2} \gamma^{-1 / \alpha} \\
& \cdot(1+\lambda T)^{-1} e^{-\lambda T-1 / 2} .
\end{aligned}
$$

Therefore, the nodes with high throughput can be further obtained.

3.2.2. Analysis of Network Energy Consumption. In wireless sensor networks, energy consumption is the key problem of data transmissions. In order to make the network energy consumption distribution more reasonable and then extend the effective work time of networks, the threshold of energy consumption of sensor nodes needs to be set, which is the foundation of node scheduling. On the basis, network lifecycle optimization can be achieved under the multiconstrained conditions.

(1) Scheduling of Node Energy Consumption. In order to avoid shortening network lifecycle due to premature exhaustion of energy of nodes, the node is not allowed to be the relay node in two cases, so two thresholds are defined accordingly.

Firstly, in all the neighbor nodes of $N_{i}$, a node $N_{j}$ can not complete the transfer task due to the low energy, shown as the following formula:

$$
\begin{aligned}
& T_{h 1}:\left(N_{j} \in \operatorname{neighbour}\left(N_{i}\right)\right) \\
& \cap\left(\operatorname{energy}\left(N_{j}\right)<\operatorname{energy}(\text { flow }),\right.
\end{aligned}
$$

where energy (flow) represents the transmission energy consumption of data flow, and neighbour $\left(N_{i}\right)$ represents the neighbor nodes of $N_{i}$, which are defined as follows:

$$
\operatorname{neighbour}\left(N_{i}\right)=\left\{N_{j}\left(N_{j} \in N\right) \cap\left(\left|N_{j}-N_{i}\right| \leq R\right)\right\},
$$

where $N$ represents the set of all nodes in the area, and $R$ represents the communication radius between nodes.

Secondly, in order to avoid inappropriate relay nodes chosen in transmission and then resulting excessive unnecessary energy consumption, the limitation of transmission energy consumption needs to be set. When node $N_{i}$ selects node $N_{j}$ for data forwarding, there is a difference between energy consumption of transmission between $N_{i}$ and $N_{j}$ and the expectation of that between $N_{i}$ and other nodes. We scale the difference by the standard deviation of energy consumption between $N_{i}$ and others and obtain the normalized difference. The probability that the normalized difference is greater than $t$ is greater than $\beta$, as shown in formula (12), which means transmission cost is too high when $N_{j}$ is taken as the relay node, and the reason for that is often due to transmission distance or interference.

$$
\begin{aligned}
& T_{h 2}: \\
& \quad P\left(\frac{\operatorname{energy}(i, j)-E(\operatorname{energy}(i, k))}{\sqrt{E\left\{(\operatorname{energy}(i, k)-E(\operatorname{energy}(i, k)))^{2}\right\}}}>t\right) \\
& \quad>\beta,
\end{aligned}
$$

where $t$ denotes the threshold set according to the scene; $\beta$ represents the probability that transmission energy consumption exceeds the setting threshold; energy $(i, j)$ represents transmission energy consumption between nodes $i$ and $j$; energy $(i, k)$ represents the transmission energy consumption between node $i$ and the other neighbor node $k ; E(\cdot)$ indicates the expectation of the transmission energy.

As mentioned above, the neighbor nodes which meet the two conditions simultaneously may be chosen to participate in the data transmission, and the purpose of these conditions is to avoid the unexpected scheduling of nodes and shorten the lifecycle of network.

(2) Network Lifecycle. Lifecycle of wireless sensor network is defined as the survival time of the first failure node caused by energy depletion, so the network lifecycle should be analyzed based on each individual. Assume that all nodes have the same initial energy and communication radius, and a fixed transmission power is used. When the distance between nodes is $d$, the transmission energy consumption with data $f$ can be expressed as below:

$$
E c(d, f)=f \cdot E_{c 1} \cdot d^{2}+f \cdot E_{c 2}
$$

where $E_{c 1}$ represents circuit power consumption of data transceiver and $E_{c 2}$ represents circuit power consumption for signal amplification during sending and receiving data.

The total amount of data forwarding between nodes $F_{i}$ can be expressed as

$$
F_{i}=\sum_{j \in \text { neighbor }\left(N_{i}\right)} f_{i j}
$$

where $f_{i j}$ represents the data flow between the current node $i$ and its neighbor node $j$.

Through the definition of node data flow and energy consumption, we can get the description of the survival time of node $i$, as shown in

$$
\operatorname{Life}_{i}=\frac{E_{i}}{\left(\left(f \cdot E c_{2}+E c(d, f)\right) \cdot \sum_{j \in \text { neighbor }\left(N_{i}\right)} f_{i j}\right)} .
$$


The lifecycle of wireless sensor network is relative to the survival time of the shortest one, so the corresponding network lifecycle of data flow $f$ is defined as

$$
\begin{aligned}
& \operatorname{Life}_{\text {net }}(f)=\min \left(\operatorname{Life}_{i}\right) \\
& \quad=\min \left(\frac{E_{i}}{\left(f \cdot E c_{2}+E c(d, f)\right) \cdot \sum_{j \in \text { neighbor }\left(N_{i}\right)} f_{i j}}\right) .
\end{aligned}
$$

Therefore, the maximum network lifecycle can be defined as

$$
\begin{aligned}
& \max \left(\operatorname{Life}_{\text {net }}(f)\right)=\max \left(\min \left(\operatorname{Life}_{i}\right)\right) \\
& =\max \left(\min \left(\frac{E_{i}}{\left(\left(f \cdot E c_{2}+E c(d, f)\right) \cdot \sum_{j \in \text { neighbor }\left(N_{i}\right)} f_{i j}\right)}\right)\right) .
\end{aligned}
$$

(3) Set of Alternative Relay Nodes. According to the above definition, we can get a set of alternative relay nodes for the transmission scheme, which is defined as follows:

$$
\begin{array}{ll}
\text { obj: } & N_{r n}=\left\{N_{j} \mid \operatorname{Dis}\left(N_{i}, N_{j}\right) \in \operatorname{scope}\left(r^{*}\right)\right\} \\
\text { st: } & \text { (1) } N_{j} \notin\left\{T_{h 1} \cup T_{h 2}\right\} ; \\
& \text { (2) } I_{\mathrm{MI}, j} \neq 0, \quad j \in \text { neighbor }\left(N_{i}\right) .
\end{array}
$$

Condition (1) requires that the relay nodes should satisfy the two thresholds of energy; condition (2) requires that the relay nodes can be aware of the information change of the target node and then track the target. Among them, $N_{r n}$ represents the set of nodes with high throughput which meets the energy threshold and is information-directed; $\operatorname{Dis}\left(N_{i}, N_{j}\right)$ represents the distance between the current node $N_{i}$ and neighbor node $N_{j}$; scope $\left(r^{*}\right)$ represents the area where the node around $r^{*}$ has high throughput. For convenience, assume that the area only contains no more than four nodes, which are closest to $r^{*}$, and the number of nodes depends on the density of neighbor nodes. Shown as Figure 3, there are six nodes; namely, $N_{1}, N_{2}, N_{3}, N_{4}, N_{5}$, and $N_{6}$ meet with the constraints, but in the valid area of $r^{*}$, only $N_{1}, N_{2}, N_{3}, N_{4}$, four nodes, at most are selected as elements of the alternative relay nodes set, and then we can choose the proper nodes for routing.

\section{Model Optimization Algorithms}

According to the transmission requirements of HS-EO, a multiagent coevolution algorithm based on self-adjusting search space is proposed. Multiagent system is an important branch in the field of distributed artificial intelligence, which is a set composed of multiple agents. The aim of that is working together to solve the problem which can not be done by single agent in the way of collaborating with each other and environments. So an important issue of the system is to use the right collaborative strategy to complete the assigned task in the process of their interaction. Multiagent system takes each agent as a unit, and each individual has its own place; through the comparison with the neighbor agents, the

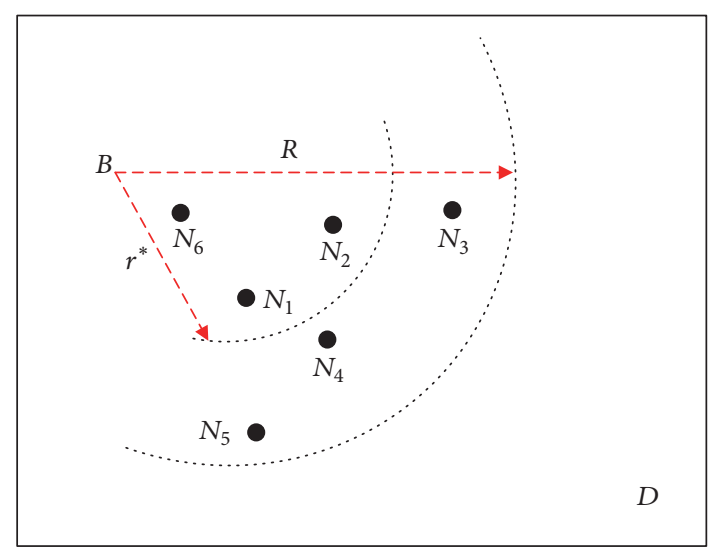

Figure 3: Selection of relay nodes.

specific behavior is carried out to achieve the population evolution, so as to obtain the process of finding optimal solutions.

HS-EO model is able to perceive all the design requirements to obtain information to track the target node and elect a collection of high throughput relay nodes under the condition of no abnormality of energy. On this basis, the transmission scheme of network life cycle maximization can be achieved. As a result, the optimization of transmission performance and overall energy consumption can be obtained. In fact, for wireless sensor networks, if without considering the meaningless consumption, energy consumption of nodes will occur only in transmission. Thus, the problem of network lifecycle maximization can be converted into a problem of lifecycle maximization for transmission path, defined as

$$
\begin{array}{ll}
\text { Obj: } & \operatorname{Max}\left(\operatorname{Life}_{\text {route }}\left(f_{j}\right)\right), \\
\text { St: } & N_{j} \in N_{r n},
\end{array}
$$

where $f_{j}$ represents the data flow through node $j$, and all the nodes in the set of alternative relay nodes are composed of alternative paths in the solution space.

4.1. Definition of Agents. Agent $\alpha$ represents a candidate solution for routing, which can be defined as the form of a multivariate vector, as shown in

$$
\alpha=\left(\alpha_{1}, \alpha_{2}, \alpha_{3}, \ldots, \alpha_{n}\right),
$$

where $\alpha_{i}$ represents the lifecycle of node $i, 0<\alpha_{i} \leq \operatorname{Life}_{i}^{\max }$, $1<i<n$, and $n$ represents the number of relay nodes. The energy possessed by agent can be expressed as follows:

$$
\operatorname{Energy}(\alpha)=\frac{1}{\max \left(\operatorname{Life}_{\text {route }}(f)\right)} \text {. }
$$

4.2. Behaviors of Agents. The key to the multiagent system is whether it is able to develop a suitable coordination strategy. The implementation of strategy is capable of generating a series of behaviors, and each agent will enhance its own energy through behaviors interacting with external. To this 
end, three kinds of behaviors are defined, namely, variation, competitive, and self-organized criticality behavior. First of all, agent can enhance its own energy by using knowledge, and variation is a kind of behavior using knowledge of itself; secondly, to obtain better solutions while computational cost is spent as little as possible, we can take the amount of calculation as limited environmental resources of agent, making each agent obtain more resources to increase its own energy by using competition. In addition, the interaction between agents often has nonlinear characteristics; in order to produce emergent phenomenon in the multiagent system, self-organized criticality behavior should be defined to improve the relationship between agents.

(1) Variation Behavior. The change of individual can promote the evolution of population, for the realization of collaborative evolution of multiagent, and variation behavior of agent is needed. The variation behavior will produce a new agent, $\alpha^{\prime}=\left(\alpha_{1}^{\prime}, \alpha_{2}^{\prime}, \ldots, \alpha_{n}^{\prime}\right)$ is used to replace the existing $\alpha=$ $\left(\alpha_{1}, \alpha_{2}, \ldots, \alpha_{n}\right)$, and the specific transformation is shown as follows:

$$
\alpha_{i}^{\prime}= \begin{cases}\alpha_{i}, & R(0,1)<\frac{1}{n}(1 \leq i \leq n) \\ r_{i}, & \text { others, }\end{cases}
$$

where $R(0,1)$ denotes a random number allocated in interval of $(0,1)$ to node $i ; r_{i}$ can get a random value, and the value belongs to $\left(0, \mathrm{Life}_{i}^{\mathrm{max}}\right]$.

(2) Competitive Behavior. Agent usually needs to compete with its neighbor nodes. Shown as Figure 4, each agent is taken as a unit, each agent will compare the energy with the surrounding neighbor nodes, and if there is no energy of neighbor nodes higher than its own, the nodes may continue to exist; otherwise, it will be replaced by other agents, and the new agent replacing the position is the individual derived by the agent with the maximum energy in the region.

Assuming that the current agent is $\alpha=\left(\alpha_{1}, \alpha_{2}, \ldots, \alpha_{n}\right)$, the agent with maximum energy is expressed as $\alpha_{\max }=\left(\alpha_{1}^{\prime}\right.$, $\left.\alpha_{2}^{\prime}, \ldots, \alpha_{n}^{\prime}\right)$. Energy comparison among agents is needed. If $\operatorname{Energy}\left(\alpha_{i, j}\right) \geq \operatorname{Energy}\left(\alpha_{\max }\right)$, then agent $\alpha=\left(\alpha_{1}, \alpha_{2}, \ldots\right.$, $\alpha_{n}$ ) does not change and continue to exist. Otherwise, $\alpha=$ $\left(\alpha_{1}, \alpha_{2}, \ldots, \alpha_{n}\right)$ will be replaced by offspring $d=\left(d_{1}, d_{2}\right.$, $\ldots, d_{n}$ ) which is produced by

$$
\begin{gathered}
d_{l} \longleftarrow r_{i} \quad R_{i}(0,1)<\rho \\
d_{l} \longleftarrow \alpha_{i}^{\prime} \quad \text { others, } \\
1 \leq i \leq n,
\end{gathered}
$$

where $r_{i}$ represents a random value that belongs to $(0$, $\left.\mathrm{Life}_{i}^{\max }\right], \rho$ is a preset value used to represent the degree of difference between vector $d=\left(d_{1}, d_{2}, \ldots, d_{n}\right)$ and $\alpha_{\max }=$ $\left(\alpha_{1}, \alpha_{2}, \ldots, \alpha_{n}\right)$, and usually the value of $\rho$ is small, so that most of the information of $\alpha_{\max }=\left(\alpha_{1}, \alpha_{2}, \ldots, \alpha_{n}\right)$ will be retained.

(3) Self-Organized Criticality Behavior. Self-organized behavior refers to the formation of the state without outside

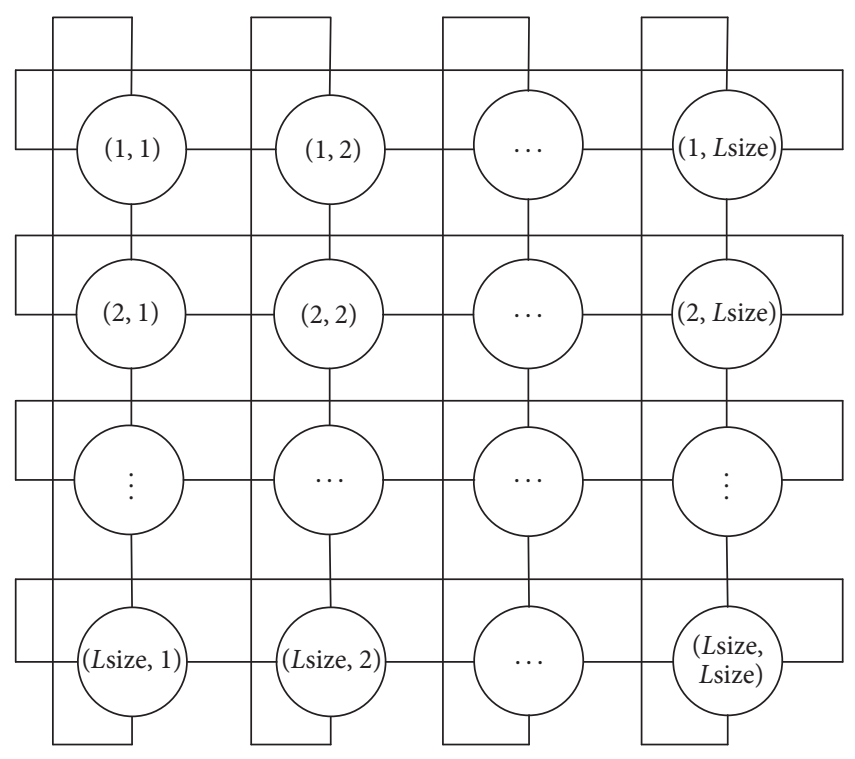

FIGURe 4: Agent grid.

intervention, produced entirely by the relationship of the occurrence of the various subsystems within the system. Criticality, as a kind of state of the system, denotes that small local changes will also affect the global situation, and all subsystem behaviors in the critical state are influencing and associated. The occurrence of the state of self-organized criticality is in accordance with the power-law distribution [26], and the definition of the behavior aims to control the individual variation and selection in population, so as to avoid stagnation and make the system update continuously.

Self-organized criticality can be described as follows: $\alpha^{t}=\left(\alpha_{1}^{t}, \alpha_{2}^{t}, \ldots, \alpha_{n}^{t}\right)$ represents the agent with the maximum energy for $t$ th generation; $P[t]$ represents power-law distribution of $t$ th generation, of which the corresponding numerical interval is $[0.2,0.8]$. The significance of this interval is that the population is in standstill when the optimal agent has no change in a number of iterations.

The steps are as follows.

Step 1 . The Agent is evolved by using variation and competitive behavior.

Step 2. If the value of $\alpha^{t}=\left(\alpha_{1}^{t}, \alpha_{2}^{t}, \ldots, \alpha_{n}^{t}\right)$ has no change in consecutive $p$ times, then go to Step 3, or Step 1 is performed.

Step 3. For each agent $\alpha=\left(\alpha_{1}, \alpha_{2}, \ldots, \alpha_{n}\right)$, if $R(0,1)<P[t]$, then a new individual will be generated to replace agent $\alpha^{t}=$ $\left(\alpha_{1}^{t}, \alpha_{2}^{t}, \ldots, \alpha_{n}^{t}\right)$ using formula (23).

4.3. Self-Adjustment of Search Space. With the number of nodes in the area increasing, leading to the expansion of the solution space, the optimal solution will be solved in larger search space, and the search speed will be reduced. In order to improve the efficiency of the algorithm, the search space needs to be converged properly. Firstly, determine the set of alternate relay nodes, in descending order according to the lifecycle. Then the $k$ nodes mentioned before will be 
selected, generally, the selected probability of which is relative high. According to the operation condition, the value of $k$ is extended dynamically, eventually extending to the whole set of alternative nodes. The step of self-adjustment for the search space is as follows.

Step 1. Initialize $k=k_{0}$; commonly the value of $k_{0}$ is small.

Step 2. Iteration is performed for the agent algorithm.

Step 3. If the optimal result is not updated in consecutive $p$ times, then go to Step 4, or Step 2 is performed.

Step 4 . Let $k \leftarrow 2 k$; then go to Step 2 .

Searching for the whole solution space directly is avoided by using the method. As a result, the optimal solution will be found quickly with high probability when the solution space is relatively large, and usually the probability of achieving the optimum can meet the requirements when $k=k_{0}$.

4.4. Solution of Routing with Optimal Lifecycle. The specific steps of the multiagent coevolution algorithm are shown below.

Step 1. Initialize agent $\alpha^{0}$; let $t \leftarrow 0$ and $T_{c} \leftarrow 0$. Initialize $A^{t}$.

Step 2. Competitive behavior is performed for each agent $\alpha^{t}$, the result of which is $\alpha_{1}^{t}$.

Step 3. If $R(0,1)<P_{v}$, variation behavior will be performed for each $\alpha_{1}^{t}$, and the corresponding result is $\alpha_{2}^{t}$.

Step 4. $A_{1}^{t *}$ will be selected from $\alpha_{1}^{t}$ to update $T_{c}$.

Step 5. If $T_{c}=p$, let $k \leftarrow 2 k$; then update $T_{c}$, or let $\alpha^{t+1} \leftarrow \alpha_{2}^{t}$, $A_{1}^{(t+1) *} \leftarrow A_{1}^{t *}$, and go to Step 8 .

Step 6. Self-organized criticality behavior is performed for each $\alpha_{2}^{t}$, and the result is $\alpha^{t+1}$.

Step 7. $A_{1}^{(t+1) *}$ is obtained from $\alpha^{t+1}$; then update $T_{c}$.

Step 8. If Energy $\left(A^{(t+1)}\right)>\operatorname{Energy}\left(A^{t}\right)$, let $A^{t+1} \leftarrow A_{1}^{(t+1) *}$, or let $A^{t+1} \leftarrow A^{t}, A_{1}^{(t+1) *} \leftarrow A^{t}$.

Step 9. $A^{t}$ will be output and the algorithm ends when the end conditions are satisfied, or let $t \leftarrow t+1$, and go to Step 2 .

$A^{t}$ represents the optimal agent update to the $t$ th generation; $A^{t *}$ represents the optimal agent in the $t$ th generation; $k$ represents the dynamic adjustment parameter; $T_{c}$ represents the times that the optimal agent keeps the same; $P_{v}$ represents the probability of performing variation behavior.

\section{Simulations and Analysis}

In order to verify the validity of the HS-EO model, a series of simulation experiments are performed on the OPNET14.5 platform. The Shortest Path First (SPF) is a classical routing
TABLE 1: Parameters of experiments.

\begin{tabular}{lc}
\hline Communication radius of nodes & 20 \\
Source coordinate & $(5,5)$ \\
Target coordinate & $(95,85)$ \\
Initial energy of nodes & $100 \mathrm{~J}$ \\
Target area & $100 \mathrm{~m} * 100 \mathrm{~m}$ \\
Test flow & $1 \mathrm{Mbit} / \mathrm{s}$ \\
Number of nodes & $50-300$ \\
\hline
\end{tabular}

method, which is simple and performs with high efficiency, and is often used in the network transmission performance testing; Minimum Energy Consumption (MEC) is the basic method of energy consumption analysis for wireless sensor network. Therefore, SPF and MEC are adopted to compare with HS-EO proposed in this paper. In addition, a variety of scenarios are set up for simulation, and indexes of hops, throughput, number of active nodes, standard deviation of residual energy, and network lifecycle are used here. The experimental parameters are set as shown in Table 1.

5.1. Hops. The hop is a basic index of routing, which affects the performance of network transmission and energy consumption and so on. 100, 200, and 300 nodes are deployed in the testing area, respectively; 30 times tests for each condition are performed. As shown in Figure 5, the value of each selected node (except for the first node) represents the average of the recent five tests. In three cases, with the number of sensor nodes in unit area increasing, hops of all methods are increased. Among them, the hops of SPF are the least. The reason of that is that the shortest path is considered, so under the same condition SPF will select path with less hops; MEC has more hops, which is due to the energy consumption considered in wireless transmission, and more hops are performed to reduce the transmission cost between point and point. In contrast, HS-EO is relatively complex, by formula (8), the node with appropriate distance is selected to communicate in order to improve the transmission performance, and so the test results show that it is between the other two methods. In particular, when the density of sensor nodes is relatively large, HS-EO has some fluctuations on hops; one of the reasons is that the interference of the node number raise makes $r^{*}$ change, and on the other hand, it is the result of considering multiple nodes scheduling to achieve the energy load balancing.

5.2. Throughput. Throughput is an important index to affect the performance of wireless sensor networks. The throughput is the effective data traffic (unit: Kbits/sec) in the case of no packet loss. Test traffic for the link is the saturation flow, and the measured values can be effective to show the performance of each method; the results of the experiment are shown as Figure 6.

When the density of nodes is low, the throughput of the three methods is not large because the selected path is less. With the number of nodes increasing in the area, throughput of each method grows in different degree. Under the 


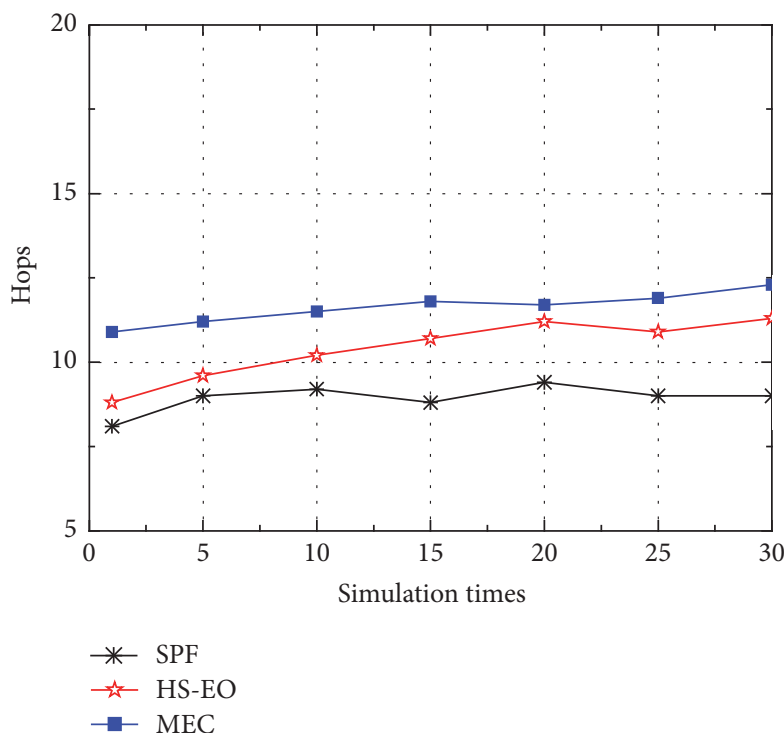

(a) 100 nodes

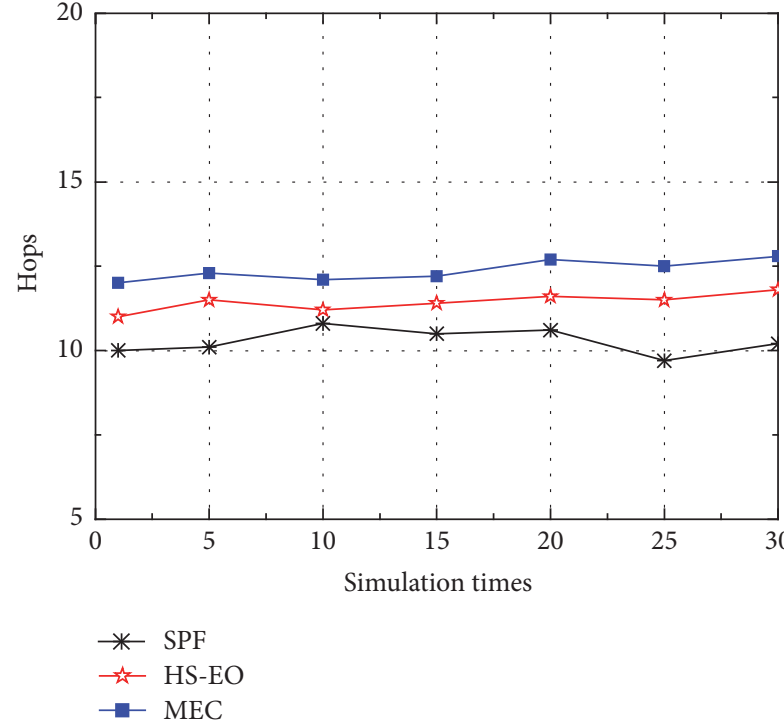

(b) 200 nodes

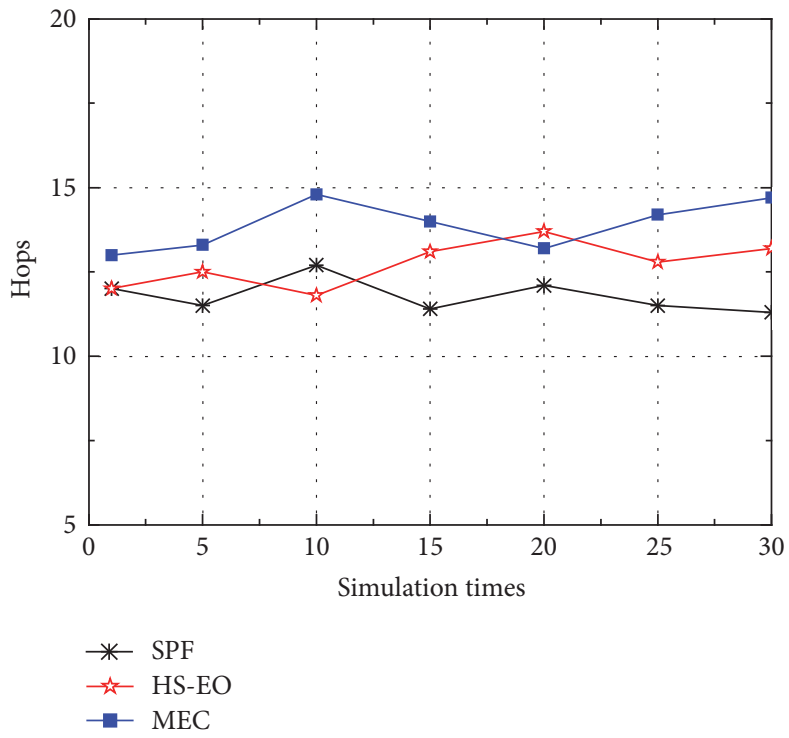

(c) 300 nodes

Figure 5: Hops analysis.

condition of same power in wireless communication, the transmission rate of long distance nodes is lower than the nodes with short distance. For SPF, although the hops reduce, the transmission distance increases accordingly, so that the test results should be lower than MEC. Compared with the previous two methods, the multiagent coevolution is adopted in HS-EO in process of tracing in order to achieve the multiobjective optimization; that is to say, to obtain load balancing between improving throughput and energy consumption of the node, nodes within the solution space are optimized for throughput, so the transmission rate is improved obviously. From the experimental data we can know that the throughput of HS-EO is consistent with its design objectives. At the same time, three kinds of methods decline with different degree after peaks in the interval $(200,250)$; this is because when the node density is too large, the interference between nodes becomes the main influencing factor of transmission, so the throughput will decrease slightly, which is related to the characteristics of wireless transmission.

5.3. Number of Activate Nodes. Active nodes refer to the nodes that are used in process of data transmission in the network lifecycle, and the number of active nodes is directly related to the performance of scheduling. If few parts of the network nodes are used with high frequency, then the energy of nodes will be depleted quickly, so as to make network enter a period in which energy fades fast. So how to make nodes schedule efficiently and uniformly is very important for improving network lifecycle and transmission performance, shown as Figure 7. 


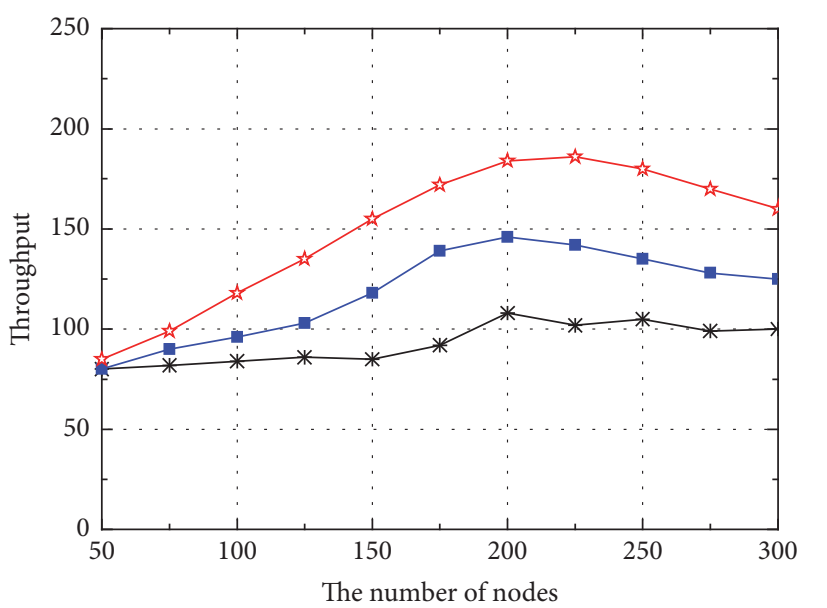

$*$ SPF
$\rightarrow$ MEC
$\rightarrow$ HS-EO

FIGURE 6: Throughput analysis.

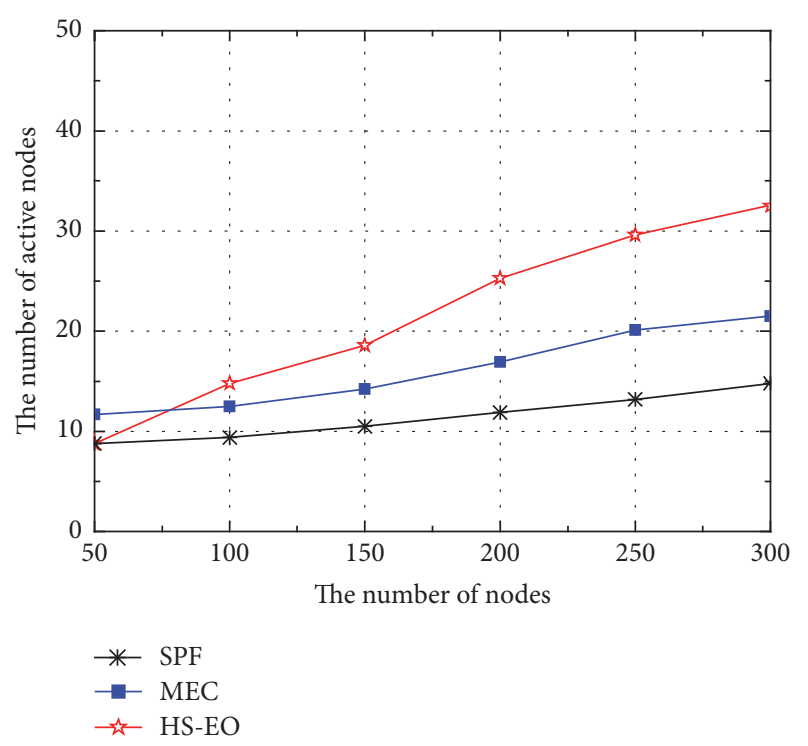

FIgURE 7: Analysis of the number of active nodes.

The number of nodes activated in SPF is only relevant to the number of relay nodes in routing process, so the number is less. Because of considering the problem of saving energy consumption for transmission end to end, MEC is capable of selecting relay node dynamically according to the energy of neighbor nodes, so there will be more nodes involved in the transmission than SPF. For HS-EO, with the increase of node density, as well as the energy threshold setting, more nodes will be chosen in scheduling due to multiagent coevolution participating in routing, so the experimental result of HS-EO is higher than that of the other two methods.

5.4. Standard Deviation of Residual Energy. Standard deviation of residual energy refers to residual energy standard deviation of other nodes when the energy of some node participating in routing is depleted, which is used to evaluate the energy consumption of the algorithm and reflects the distribution of the residual energy of each node. If the residual energies of all nodes are close to each other, namely, the standard deviation is smaller, this indicates that the network energy consumption distribution is uniform; otherwise, the energy distribution is not uniform and may lead to network lifecycle shortening. Standard deviation of residual energy can be defined as

$$
\begin{aligned}
\sigma_{E} & =\frac{\sqrt{E\left\{\left[E_{i}-E\left(E_{i}\right)\right]^{2}\right\}}}{E_{\mathrm{ini}}}, \quad i \in n \\
E\left(E_{i}\right) & =\frac{\sum_{i=1}^{n} E_{i}}{n}
\end{aligned}
$$

where $n$ represents the total number of sensor nodes in network, $E_{i}$ represents the current residual energy of node $i, E_{\text {ini }}$ represents the initial energy value of nodes, and $E\left(E_{i}\right)$ represents the average residual energy of $n$ nodes.

To test the distribution of energy consumption of nodes, 30 times tests are performed in the case of 200 nodes, as shown in Figure 8. SPF fails to schedule the network energy consumption effectively, so the test result is relatively high and large fluctuations appear. MEC takes into account the optimization of transmission energy consumption of relay nodes and adjusts according to the energy consumption of neighbor nodes, so the value of $\sigma_{E}$ is more uniform than SPF. Among the three methods, standard deviation of residual energy in HS-EO is the lowest, which reflects that the method can make load sharing of energy consumption more uniform. The reasons are as follows: firstly, threshold scheduling can avoid the extremes of energy consumption of nodes, thereby reducing the possibility of standard deviation being too large; secondly, because the purpose of multiagent coevolution is to select the node which has better energy in the alternative set of nodes and achieve the optimization of lifecycle for link, there is no significant difference of standard deviation among the residual energies of nodes; namely, the value of that is not high; thirdly, the experimental results of number of active nodes show that the number of relay nodes involved in HS-EO is more than the other two methods; therefore the sample space is large and the measurement data are more accurate. Thereby, in the aspects of energy scheduling, HSEO is superior to the other two methods.

5.5. Network Lifecycle. Network lifecycle is an important index to measure the effectiveness of nodes. We test 50 times in the case of 200 nodes, and the experimental results are shown in Figure 9. For SPF, because the relay nodes are relatively small, the distribution of energy is not uniform and fails to optimize the communication cost as well, so the lifecycle of network is relatively short. MEC adopts the strategy with the low energy consumption; at the same time, the experimental results of standard deviation of residual energy show that the distribution of energy consumption is more uniform than $\mathrm{SPF}$, and therefore the network lifecycle is relatively long. For HS-EO, although high throughput transmission will bring the energy loss partly, multiple nodes can be scheduled with 


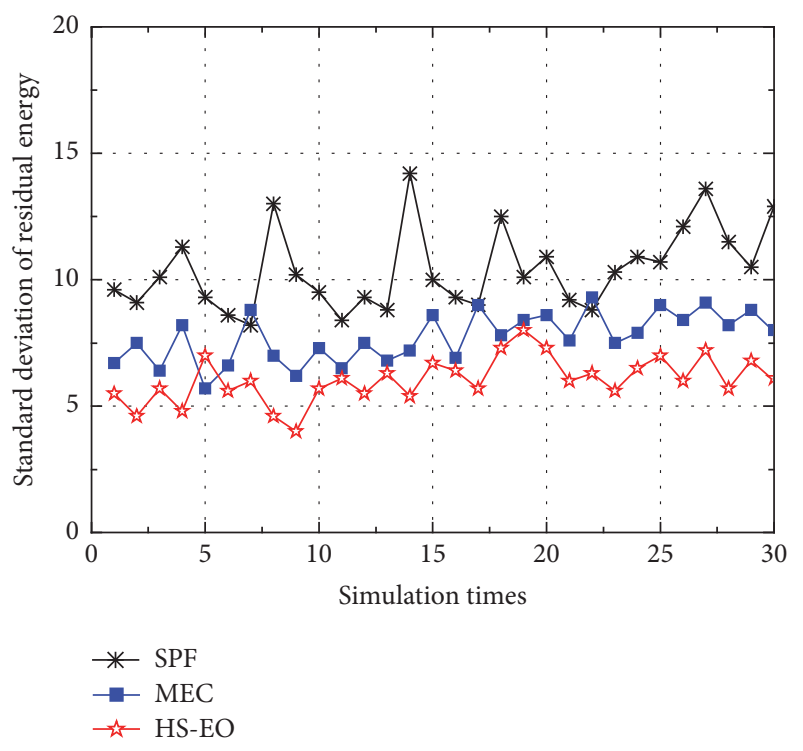

FIgURE 8: Analysis of standard deviation of residual energy.

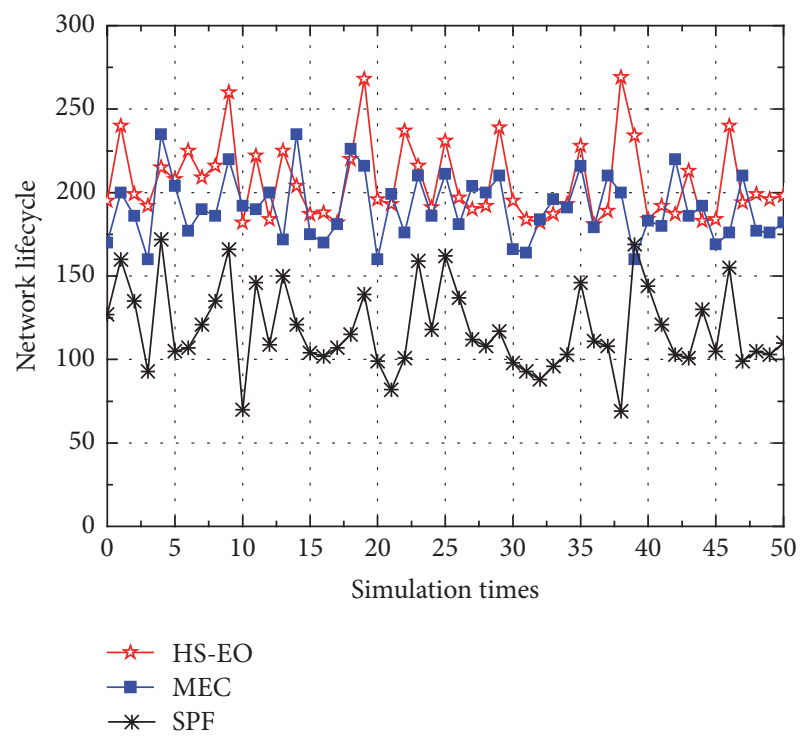

FIgURE 9: Analysis of network lifecycle.

energy thresholds used in multiagent coevolution, so as to balance the energy consumption and prolong the network lifecycle timely.

\section{Conclusions}

In wireless sensor network, the research of target tracing always focuses on object recognition, positioning, tracing, and other issues, whereas there are no further studies on routing methods. In view of the situation, according to the current needs of energy saving and high speed transmission for wireless sensor network, a high speed transmission and energy optimization model HS-EO oriented to lifecycle maximization is proposed in the paper. Meanwhile multiagent coevolution is adopted to implement optimization for maximum lifecycle. Through the comparison with SPF and MEC, in the case of five different indexes, the results show that HS-EO can improve the transmission rate effectively, prolong the network lifecycle, and enhance the performance of the network as a whole. In further studies, we will combine network function virtualization to do more related researches.

\section{Competing Interests}

The authors declare that they have no competing interests.

\section{Acknowledgments}

This work is supported by the National Natural Science Foundation of China (61403109) and the Scientific Research Fund of Heilongjiang Provincial Education Department (12541169).

\section{References}

[1] I. F. Akyildiz, W. L. Su, and Y. Sankarasubramaniam, "A sensor grid: coverage, connectivity and diameter," in Proceedings of the IEEE INFOCOM, pp. 1073-1083, San Francisco, Calif, USA, 2003.

[2] D. Culler, D. Estrin, and M. Srivastava, "Overview of sensor networks," Computer, vol. 37, no. 8, pp. 41-49, 2004.

[3] O. Salem, A. Guerassimov, A. Mehaoua, A. Marcus, and B. Furht, "Sensor fault and patient anomaly detection and classification in medical wireless sensor networks," in Proceedings of the IEEE International Conference on Communications (ICC '13), pp. 4373-4378, IEEE, Budapest, Hungary, June 2013.

[4] T.-D. Nguyen, T. T. Thanh, L.-L. Nguyen, and H.-T. Huynh, “On the design of energy efficient environment monitoring station and data collection network based on ubiquitous wireless sensor networks," in Proceedings of the 2015 International Conference on Computing and Communication Technologies: Research, Innovation, and Vision for Future (IEEE RIVF '15), pp. 163-168, Can Tho, Vietnam, January 2015.

[5] G. Owojaiye and Y. Sun, "Focal design issues affecting the deployment of wireless sensor networks for intelligent transport systems," Intelligent Transport System, vol. 6, no. 4, pp. 421-432, 2012.

[6] R. Gupta, K. Sultania, P. Singh, and A. Gupta, "Security for wireless sensor networks in military operations," in Proceedings of the 4th International Conference on Computing, Communications and Networking Technologies (ICCCNT '13), July 2013.

[7] J. Yick, B. Mukherjee, and D. Ghosal, "Wireless sensor network survey," Computer Networks, vol. 52, no. 12, pp. 2292-2330, 2008.

[8] D. Quintas and V. Friderikos, "Minimum energy and maximum capacity paths in multi hop wireless networks," in Proceedings of the IEEE Conference on Green Communications (GreenCom '11), pp. 30-35, IEEE, New York, NY, USA, September 2011.

[9] C. Bae and W. E. Stark, "Minimum energy-per-bit wireless multi-hop networks with spatial reuse," Journal of Communications and Networks, vol. 12, no. 2, pp. 103-113, 2010.

[10] T. N. Minh, "Minimizing energy consumption in random walk routing for wireless sensor networks utilizing compressed sensing," in Proceedings of the 8th International Conference on System of Systems Engineering, pp. 297-301, Maui, Hawaii, USA, June 2013. 
[11] Y. Liu, C. Chen, H. Yu, and X. Guan, "Distortion analysis for delay tolerant data collection for high-speed wireless sensor and actor networks," in Proceedings of the 10th World Congress on Intelligent Control and Automation (WCICA '12), pp. 4452-4457, July 2012.

[12] U. S. Visweswaraiya and K. S. Gurumurthy, "A novel, dynamic data dissemination [D3] technique for congestion avoidance/ control in high speed wireless multimedia sensor networks," in Proceedings of the 2013 5th International Conference on Computational Intelligence, Modelling and Simulation (CIMSim '13), pp. 351-356, Seoul, Republic of Korea, September 2013.

[13] H. R. Karkvandi, E. Pecht, and O. Yadid-Pecht, "Effective lifetime-aware routing in wireless sensor networks," IEEE Sensors Journal, vol. 11, no. 12, pp. 3359-3367, 2011.

[14] H. Wang, N. Agoulmine, M. Ma, and Y. Jin, "Network lifetime optimization in wireless sensor networks," IEEE Journal on Selected Areas in Communications, vol. 28, no. 7, pp. 1127-1137, 2010.

[15] C. Song, M. Liu, J. Cao, Y. Zheng, H. Gong, and G. Chen, "Maximizing network lifetime based on transmission range adjustment in wireless sensor networks," Computer Communications, vol. 32, no. 11, pp. 1316-1325, 2009.

[16] Y.-Y. Li, H.-Y. Zhang, and Y.-H. Liu, "Energy saving target tracking using mobile sensor networks," Zidonghua Xuebao/Acta Automatica Sinica, vol. 33, no. 11, pp. 1156-1162, 2007.

[17] W. Liang, H.-B. Yu, C.-Z. Zang, and Z.-H. Xing, "Research on simulation platform of Wireless Sensor Network based on multi-agent," Journal of System Simulation, vol. 18, no. 4, pp. 913937, 2006.

[18] C. Che and W. Liang, "Cooperation problem of wireless sensor network based on multi-agent," Chinese Journal of Scientific Instrument, pp. 229-236, 2005.

[19] C. Z. Zang and W. H. Liang, "Target tracking based on moving agent in wireless sensor network," Control Theory \& Applications, pp. 293-301, 2006.

[20] M. Dong, K. Ota, L. T. Yang, S. Chang, H. Zhu, and Z. Zhou, "Mobile agent-based energy-aware and user-centric data collection in wireless sensor networks," Computer Networks, vol. 74, pp. 58-70, 2014.

[21] C.-H. Kuo, T.-S. Chen, and Y.-H. Lo, "Efficient traffic load reduction algorithms for mitigating query hotspots for wireless sensor networks," International Journal of Ad Hoc and Ubiquitous Computing, vol. 18, no. 3, pp. 153-163, 2015.

[22] W. Z. Guo, N. X. Xiong, H.-C. Chao, S. Hussain, and G. Chen, "Design and analysis of self-adapted task scheduling strategies in wireless sensor networks," Sensors, vol. 11, no. 7, pp. 65336554, 2011.

[23] X. Wang, D.-W. Bi, L. Ding, and S. Wang, "Agent collaborative target localization and classification in wireless sensor networks," Sensors, vol. 7, no. 8, pp. 1359-1386, 2007.

[24] R. S. Dina and M. F. Khaled, "Steiner-tree based informationdirected routing," in Proceedings of the International Conference on Computer Engineering, pp. 62-67, 2010.

[25] J.-L. Hsu and I. Rubin, "On routing and rate control strategies in wireless multi-hop random access networks," in Proceedings of the IEEE International Conference on Communications (ICC '07), pp. 3395-3400, June 2007.

[26] T. Krink, R. Thomsen, and P. Rickers, "Applying self-organised criticality to evolutionary algorithms," in Parallel Problem Solving from Nature PPSN VI: 6th International Conference Paris, France, September 18-20, 2000 Proceedings, vol. 1917 of Lecture Notes in Computer Science, pp. 375-384, Springer, Berlin, Germany, 2000. 


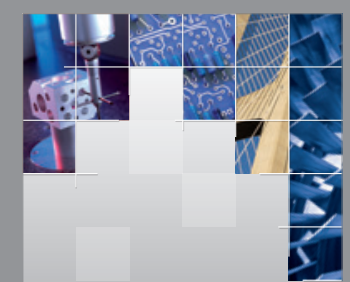

\section{Enfincering}
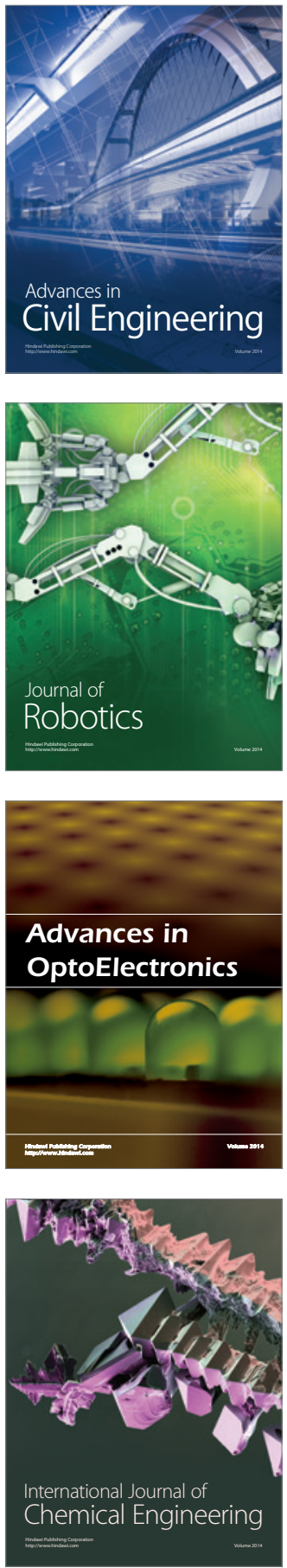

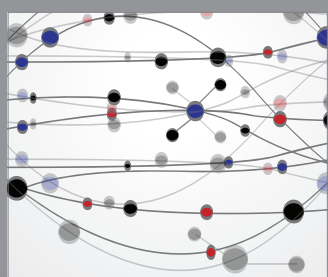

The Scientific World Journal

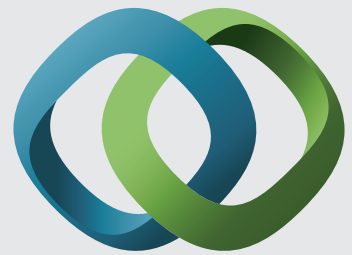

\section{Hindawi}

Submit your manuscripts at

http://www.hindawi.com
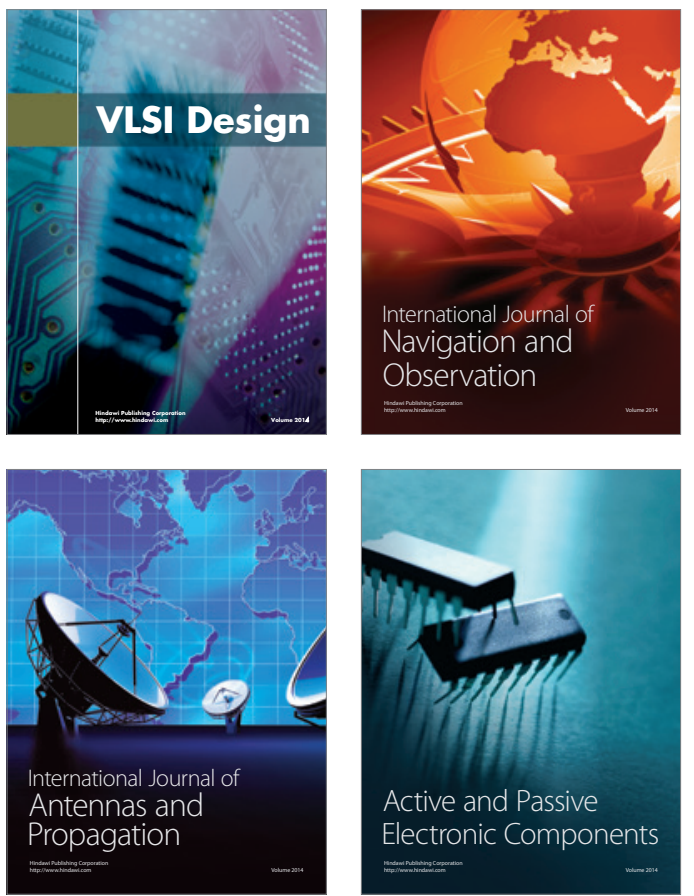
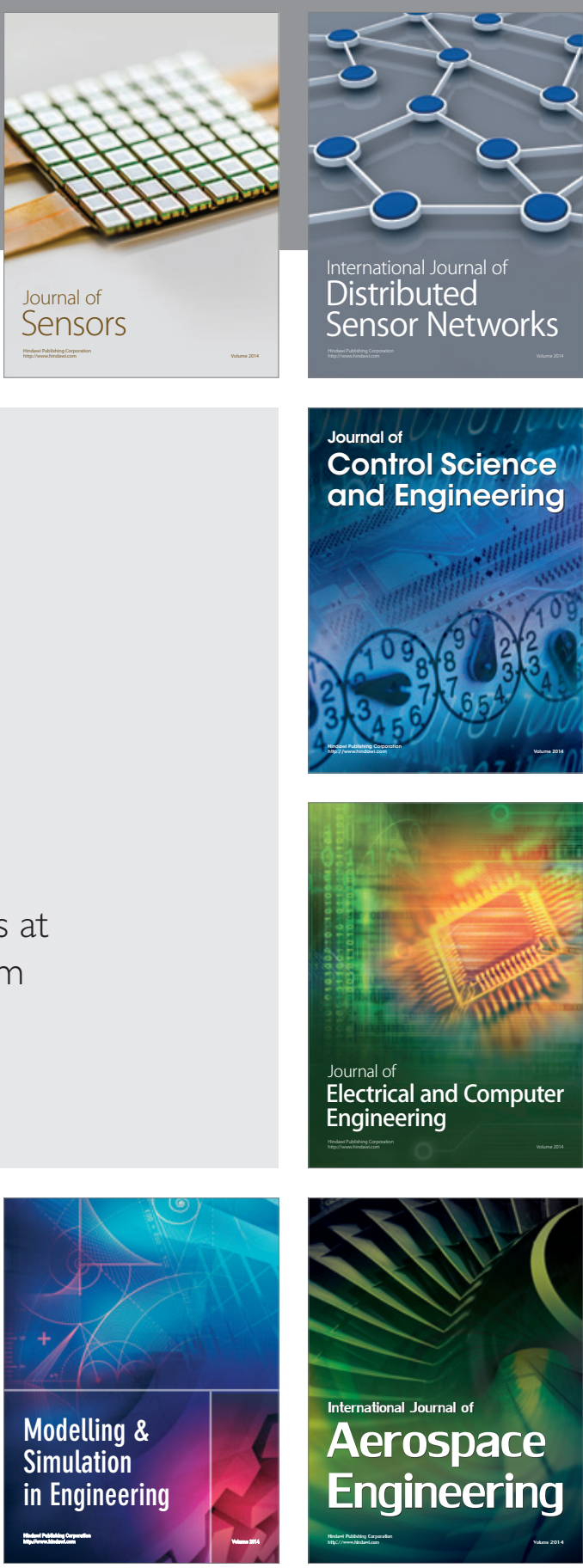

International Journal of

Distributed

Sensor Networks

Journal of

Control Science

and Engineering
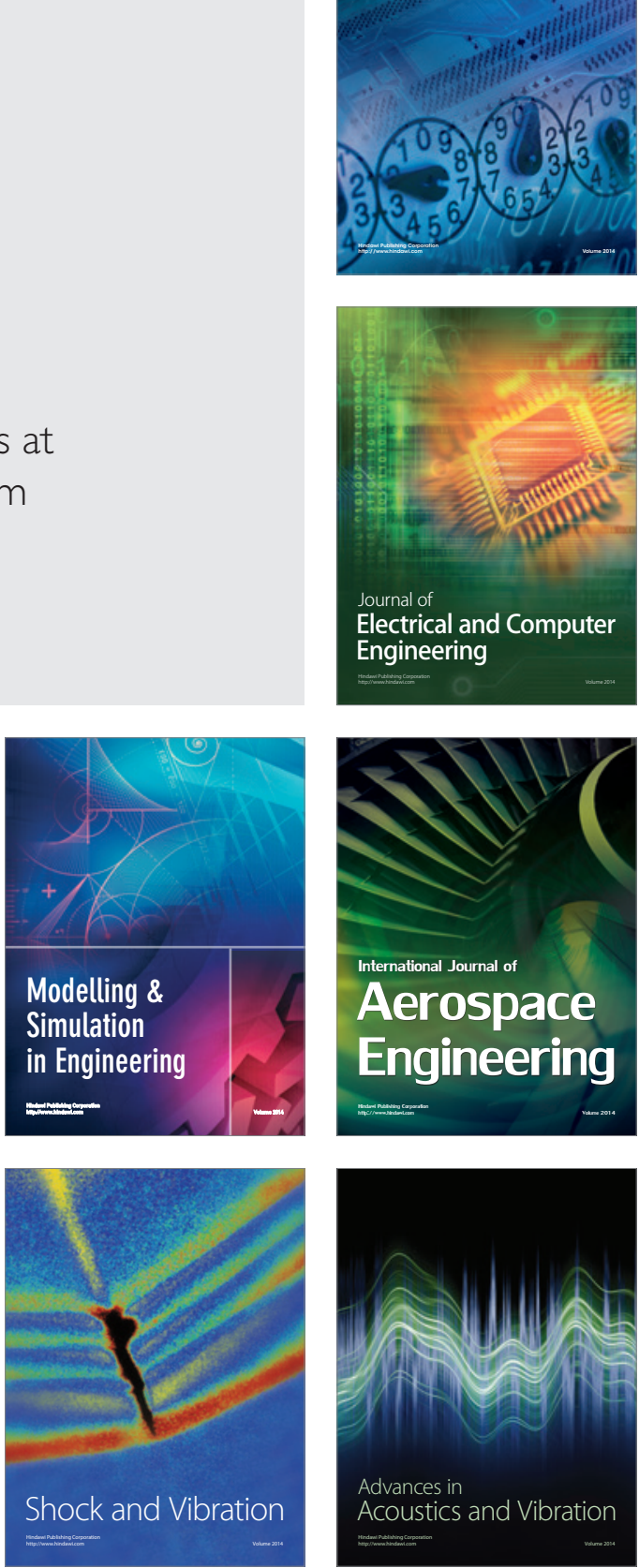